\title{
Clinical characteristics and outcomes of adult patients with acquired thrombotic thrombocytopenic purpura: a single center retrospective study
}

\author{
Fang Huang ${ }^{1 \#}$, Xiao-Ping $\mathrm{Li}^{1 \#}$, Ying $\mathrm{Xu}^{1 \#}$, Xin-Yue $\mathrm{Li}^{1}$, Dong-Rong $\mathrm{Li}^{1}$, Yan Hao ${ }^{1}, \mathrm{Zi}-\mathrm{Qiang} \mathrm{Yu}^{2}, \mathrm{Jun}^{\mathrm{Jin}}{ }^{1}$, \\ Jun Wang ${ }^{1 \wedge}$
}

${ }^{1}$ Department of Intensive Care Medicine, The First Affiliated Hospital of Soochow University, Suzhou, China; ${ }^{2}$ Department of Hematology, The First Affiliated Hospital of Soochow University, Suzhou, China

Contributions: (I) Conception and design: F Huang, XP Li, ZQ Yu, J Jin, J Wang; (II) Administrative support: J Wang; (III) Provision of study materials or patients: F Huang, Y Xu, DR Li, XP Li, Y Hao, J Jin, J Wang; (IV) Collection and assembly of data: Y Xu, XP Li, DR Li, XY Li, Y Hao; (V) Data analysis and interpretation: XP Li, J Wang; (VI) Manuscript writing: All authors; (VII) Final approval of manuscript: All authors.

\#These authors contributed equally to this work.

Correspondence to: Jun Wang. Department of Intensive Care Medicine, The First Affiliated Hospital of Soochow University, No. 188 Shizi Street, Suzhou 215006, China. Email: dr_wangjun@suda.edu.cn.

Background: Thrombotic thrombocytopenic purpura (TTP) is a rare disease and a potentially lifethreatening thrombotic microangiopathy. Although the diagnostic and therapeutic techniques have improved, it is still difficult for clinicians to identify early due to different initial clinical manifestations and the incidence and survival rate are reported inconsistently. This study investigated the clinical characteristics, treatment strategies, and treatment outcomes of adult patients with acquired TTP.

Methods: A retrospective analysis of 55 patients (35 females and 20 males) treated for acquired TTP from January 1, 2013 to December 31, 2017 was conducted. The analysis included clinical manifestations at onset, treatment efficacy measures, survival, cause of death, and the APACHE II (Acute Physiology and Chronic Health Evaluation II) and SOFA (sequential organ failure assessment) scores.

Results: At onset, in addition to thrombocytopenia and hemolysis, 50 patients (90.91\%) presented with neurological abnormalities, but only 19 (34.55\%) showed the classic TTP pentad of symptoms. The overall mortality rate was $34.55 \%$. Plasma exchange (PEX) was performed in 49 patients. The most effective treatment was PEX with a normal dose of corticosteroid and rituximab which showed a response rate of $81.25 \%$. The main cause of death was cerebral hemorrhage. The APACHE II and SOFA scores were higher in non-survivors compared to survivors (APACHE II: $20.12 \pm 7.83$ vs. $11.50 \pm 4.49, \mathrm{P}<0.05$; SOFA: $12.06 \pm 3.27$ vs. 7.74 $\pm 2.10, \mathrm{P}<0.05)$. Non-survivors had higher levels of lactic dehydrogenase (LDH; $1,646.94 \pm 1,269.48$ vs. $942.76 \pm 740.58 \mathrm{IU} / \mathrm{L}, \mathrm{P}=0.015)$, and higher numbers of schistocytes $(6.18 \% \pm 4.69 \%$ vs. $3.44 \% \pm 3.13 \%$, $\mathrm{P}=0.035$ ) compared to survivors.

Conclusions: TTP progressed rapidly, and its clinical manifestations varied between patients. The diagnosis depended on the clinical features and laboratory tests. Combination therapy with PEX, immunosuppressive therapy, and rituximab may be useful. Higher APACHE II and SOFA scores, higher LDH levels, and a greater degree of schistocytosis were associated with the severity and outcome of TTP.

Keywords: Thrombotic thrombocytopenic purpura (TTP); plasma exchange (PEX); Acute Physiology and Chronic Health Evaluation II APACHE II); sequential organ failure assessment (SOFA)

Submitted Mar 01, 2021. Accepted for publication Apr 28, 2021

doi: 10.21037/apm-21-759

View this article at: http://dx.doi.org/10.21037/apm-21-759

\footnotetext{
$\wedge$ ORCID: 0000-0001-8708-3096.
} 


\section{Introduction}

Acquired thrombotic thrombocytopenic purpura (TTP) is a potentially life-threatening thrombotic microangiopathy. Its clinical manifestations include thrombocytopenia and hemolytic anemia, which can lead to multi-organ failure (1). It is a rare disease with an annual incidence estimated at 4-11 cases per million people in the United States (2). The typical clinical presentation of acquired TTP includes the "classic" pentad of symptoms, namely, microangiopathic hemolytic anemia, thrombocytopenia, neurologic abnormalities, renal disease, and fever (3). Among these symptoms, microangiopathic hemolytic anemia, thrombocytopenia, and neurologic abnormalities are required for the diagnosis (the classic triad).

TTP is caused by acquired or congenital severe deficiency of the von Willebrand factor regulatory enzyme, ADAMTS13 (a disintegrin and metalloproteinase with a thrombospondin type 1 motif, member 13) (4-6). In patients with acquired TTP, decreased ADAMTS13 activity leads to the uncontrolled formation of von Willebrand factorrich platelet thrombi in the microvasculature (7), which causes tissue ischemia and organ dysfunction (including the heart, kidneys, and brain), and results in long-term complications (such as cognitive deficits, depression, and arterial hypertension) and early death (8-12).

In the past few decades, with the introduction of new therapies, the survival rate for TTP patients has significantly increased. The standard treatment for patients with acquired TTP consists of plasma exchange (PEX) and immunosuppressive therapy. PEX can remove the autoantibodies and von Willebrand factor multimers, and restore ADAMTS13 activity in the plasma, while immunosuppressive therapy inhibits the formation of new autoantibodies $(1,13)$. If PEX cannot be conducted, plasma infusion at up to $30 \mathrm{~mL} / \mathrm{kg}$ daily can be performed as a replacement measure, provided the patient can tolerate the fluid load (14).

The present study investigated the clinical characteristics and treatment outcomes of 55 adult patients with TTP who were treated in a university hospital in China.

We present the following article in accordance with the STROBE reporting checklist (available at http://dx.doi. org/10.21037/apm-21-759).

\section{Methods}

\section{Study population and setting}

A retrospective medical record analysis of consecutive patients diagnosed with acquired TTP in the First Affiliated Hospital of Soochow University for a period of five years (from January 1, 2013 to December 31, 2017) was performed. The diagnostic criteria included typical clinical manifestations and decreased ADAMTS13 activity. The exclusion criterion was PEX treatment obtained before hospitalization in our institution. During this period, 68 patients were diagnosed with TTP and hospitalized, but 13 patients were excluded according to the exclusion criteria, and the final study population consisted of a total of 55 cases (35 females and 20 males).

\section{Diagnosis of TTP}

\section{Laboratory work-up}

The laboratory work-up at admission included platelet count (Plt), hematocrit (HCT), schistocytes, lactic dehydrogenase (LDH) levels, fibrinogen (Fbg), activated partial thromboplastin time (APTT), total bilirubin (TBIL), and serum creatinine $(\mathrm{Cr})$.

\section{Testing for ADAMTS13 activity and functional inhibitors}

ADAMTS13 activity was assessed in the citrated plasma collected before the initial PEX by residual collagen binding assay (R-CBA) (15). ADAMTS13 deficiency was defined as ADAMTS13 activity below 5\% (16). ADAMTS 13 functional inhibitor activity was measured using the fluorescence resonance energy transfer (FRET) method. The result was considered negative when the inhibitor titers were below 0.4 Bethesda units $(\mathrm{BU} / \mathrm{mL})$ and positive when the titers were over $2.0 \mathrm{BU} / \mathrm{mL}$. Results that ranged between $0.4-2.0 \mathrm{BU} / \mathrm{mL}$ were considered inconclusive.

\section{PEX treatment}

PEX was performed at a rate of $40 \mathrm{~mL} / \mathrm{kg}$ per day using a Prisma-flex (GAMBRO) device. If there was insufficient fresh frozen plasma (FFP), at least 2,000 $\mathrm{mL}$ of plasma was exchanged daily. In 5 patients, PEX treatment was conducted once with only $2,000 \mathrm{~mL}$ of plasma because of shortage of plasma supply, and the other PEX treatments were performed at the normal rate.

\section{Other treatments}

In addition to PEX, all subjects received immunosuppressive therapy. In all cases, the immunosuppressive therapy 
Table 1 Etiology of patients with acquired thrombotic thrombocytopenic purpura (TTP) in the study cohort

\begin{tabular}{lc}
\hline Etiological factor & Number of patients (\%) \\
\hline Autoimmune disease $^{\mathrm{a}}$ & $5(9.09)$ \\
Infection $^{\mathrm{b}}$ & $5(9.09)$ \\
Tumor $^{\mathrm{c}}$ & $3(5.45)$ \\
Pregnancy & $2(3.64)$ \\
Drug & $1(1.82)$ \\
Unknown & $39(70.91)$
\end{tabular}

a , includes sicca syndrome $(n=3)$, systemic lupus erythematosus $(n=1)$, and rheumatoid arthritis $(n=1) ;{ }^{b}$, includes pneumonia $(n=3)$, enteritis $(n=1)$, and sepsis $(n=1) ;{ }^{c}$, includes breast cancer $(n=1)$, cervical cancer $(n=1)$, and blood tumor $(n=1)$.

was based on methylprednisolone in personalized doses. Some patients received pulse corticosteroid therapy with more than $240 \mathrm{mg}$ of methylprednisolone (240-500 mg) per day at the early stage, which was subsequently reduced to the normal dose. Other patients were given a normal dose ( $80 \mathrm{mg}$ methylprednisolone) every day from onset. Rituximab was administered in patients with the ADAMTS13 functional inhibitor $\left(375 \mathrm{mg} / \mathrm{m}^{2}\right.$, once weekly for 4 consecutive weeks). In addition, antibiotics, shock therapy (for example, fluid resuscitation in response to hemodynamic monitoring), mechanical ventilation, and renal replacement treatment were administered as required.

\section{Clinical outcomes}

Response was defined as the normalization of Plt $\left(100 \times 10^{9} / \mathrm{L}\right.$ or higher, and re-test results not lower than $100 \times 10^{9} / \mathrm{L}$ within 48 hours) and the improvement of clinical symptoms. Exacerbation was defined as recurrent thrombocytopenia after achieving a response. Remission was defined as a sustained response for more than 30 days after PEX was terminated. Relapse was defined as the recurrence of an acute episode of TTP after remission.

\section{Statistical analysis}

Statistical analyses were performed using SPSS 17.0 software. All normally distributed data were expressed as mean \pm standard deviation (SD). Comparisons between different groups were performed using Student's $t$-test. All categorical variables were expressed in frequency and compared using $\chi^{2}$-test or continuous correction Chi-square test. The survival rate was analyzed in treatment subgroups using the Kaplan-Meier survival curve. All tests were twosided, and for all statistical analyses, statistical significance was set at $\mathrm{P}<0.05$.

\section{Ethical statement}

This study was reviewed and approved by the Ethics Committee of The First Affiliated Hospital of Soochow University. All procedures performed in studies involving human participants were in accordance with the ethical standards of the institutional and national research committee and in accordance with the Helsinki Declaration (as revised in 2013). Written informed consent was obtained from all participating patients.

\section{Results}

\section{Patients}

A total of 55 patients ( 35 females and 20 males), who were diagnosed with acquired TTP between January 1 , 2013 and December 31, 2017, were enrolled into the retrospective analysis. The average age of the patients was $43.73 \pm 14.87$ years. There was no significant age difference in the male and female cohorts $(45.75 \pm 13.89 v s .42 .57 \pm$ 15.48 years, $\mathrm{P}=0.451$ ). The APACHE II (Acute Physiology and Chronic Health Evaluation II) score was higher in the female group $(15.02 \pm 7.34$ in females $v s .12 .65 \pm 6.05$ in males) but this was not statistically significant $(\mathrm{P}=0.225)$.

\section{Etiological factors}

The etiological factors were detected in 16 patients $(29.09 \%$, Table 1). The most common causes of TTP were infection and autoimmune disease ( $\mathrm{n}=5,9.09 \%$ each), followed by tumor $(\mathrm{n}=3,5.45 \%)$, pregnancy $(\mathrm{n}=2,3.64 \%)$, and drugs (dehydroandrograpolide succinate injection) ( $\mathrm{n}=1,1.82 \%$ ). No predisposing causes were found in 39 patients $(70.91 \%)$.

\section{Clinical features}

All patients presented with thrombocytopenia. Significant bleeds were observed in 30 patients. The most common bleeding site was the skin, and bleeding was accompanied by ecchymosis. Clinical features of 55 patients with acquired thrombotic thrombocytopenic purpura were described in Table 2. In 19 patients (34.55\%), the classic pentad of 
Table 2 Clinical features of patients with acquired thrombotic thrombocytopenic purpura (TTP)

\begin{tabular}{lc}
\hline Clinical symptom & Number of patients (\%) \\
\hline Thrombocytopenia & $55(100.00)$ \\
Hemolysis & $55(100.00)$ \\
Neurological abnormalities & $50(90.91)$ \\
Headache and vertigo & $26(47.27)$ \\
Aphasia & $6(10.91)$ \\
Delirium & $9(16.36)$ \\
Disturbance of consciousness & $41(75.55)$ \\
Seizures & $28(50.91)$ \\
Fever & $39(70.91)$ \\
Renal failure & $32(58.18)$ \\
\hline
\end{tabular}

Table 3 Treatment modalities and response rates in patients with acquired thrombotic thrombocytopenic purpura (TTP)

\begin{tabular}{|c|c|c|}
\hline Treatment modality & $\begin{array}{c}\text { Number of } \\
\text { patients }\end{array}$ & $\begin{array}{c}\text { Response } \\
\text { rate (\%) }\end{array}$ \\
\hline No PEX & 6 & $3(50.00)$ \\
\hline PEX & 49 & $33(67.35)$ \\
\hline PEX + pulse corticosteroid ${ }^{a}$ & 12 & $6(50.00)$ \\
\hline PEX + corticosteroid ${ }^{\mathrm{b}}$ & 21 & $14(66.67)$ \\
\hline PEX + corticosteroid + rituximab & 16 & $13(81.25)$ \\
\hline
\end{tabular}

a , methylprednisolone pulses in doses exceeding 240-500 mg daily; ${ }^{b}$, methylprednisolone doses of $80 \mathrm{mg}$ daily. PEX, plasma exchange.

symptoms was observed, while in 5 patients (9.09\%), merely the triad was present (thrombocytopenia, fever, and renal disease). In 5 patients (9.09\%), only 2 classic symptoms were detected (thrombocytopenia and hemolysis). The first symptoms at onset were neurological abnormalities (19 cases), thrombocytopenia (15 cases), and hemolysis (5 cases).

\section{Treatments and outcome}

PEX was administered to 49 patients. A total of 3 patients were not eligible for PEX treatment due to the severity of their condition and rapid progression to death. A total of 3 recurrent patients responded to corticosteroid therapy and did not require PEX [the platelet counts (Plt) of these three

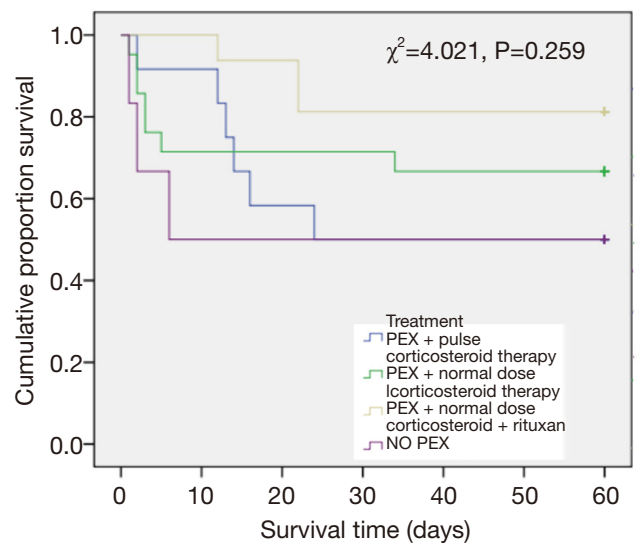

Figure 1 The cumulative proportion survival of patients with acquired thrombotic thrombocytopenic purpura according to the different treatment protocols. PEX, plasma exchange.

patients increased rapidly]. The median number of PEX procedures was 6 per hospitalization (range, 2-29). The median plasma volume for PEX was 11,500 mL. All patients in the study group received corticosteroid therapy.

In the subgroup of patients who received PEX, 12 subjects were treated with pulse corticosteroid therapy (240-500 mg methylprednisolone for 1 day per patient), 21 patients were administered a regular dose of corticosteroid therapy (a total dose of $80 \mathrm{mg}$ methylprednisolone for 1 day per patient), and the remaining 16 patients were given corticosteroids and rituximab. The response rate of patients treated with PEX combined with a normal dose corticosteroid and rituximab (81.25\%) was higher than the response rates in the other two groups. The response rate of patients treated with pulse dose corticosteroid (50\%) was the lowest among the treatment groups. However, there were no significant differences between these 3 different treatments. The specific data is listed in Table 3. There were no significant differences in patient survival among these treatments (Figure 1, $\chi^{2}=4.021, \mathrm{P}=0.259$ ).

A total of 19 patients died in the present study, and the overall mortality was $34.55 \%$. The main cause of death was cerebral hemorrhage $(n=12,63.16 \%)$. The specific causes of death are listed in Table 4. Infection was a common side effect of pulse corticosteroid, with 2 patients in this treatment group presenting with infections. The precise cause of death could not be confirmed in 3 patients and since no clear clinical indications were observed, these patients did not undergo autopsies.

The APACHE II and sequential organ failure assessment 
(SOFA) scores were higher in non-survivors compared to survivors (APACHE II: $20.12 \pm 7.83$ vs. $11.50 \pm 4.49, \mathrm{P}<0.05$; SOFA: $12.06 \pm 3.27$ vs. $7.74 \pm 2.10, \mathrm{P}<0.05)$. Similarly, nonsurvivors had higher levels of LDH $(1,646.94 \pm 1,269.48$ vs. $942.76 \pm 740.58 \mathrm{IU} / \mathrm{L}, \mathrm{P}<0.05)$, and a higher degree of schistocytosis $(6.18 \% \pm 4.69 \%$ vs. $3.44 \% \pm 3.13 \%, \mathrm{P}<0.05)$ compared to survivors. No significant differences in age, Plt, HCT, TBIL, Cr, and APTT were observed between survivors and non-survivors. Similarly, no statistical difference in time from diagnosis to the initial PEX treatment was observed between survivors and nonsurvivors (Table 5). Gender did not correlate with the outcome. None of the clinical symptoms correlated with the treatment outcome. It is noteworthy that patients presenting with disturbances of consciousness and patients with the classic pentad syndrome and pneumonia had a lower survival rate, but the differences were not statistically

Table 4 Cause of death in patients with acquired thrombotic thrombocytopenic purpura (TTP)

\begin{tabular}{lc}
\hline Cause of death & Number of patients (\%) \\
\hline Cerebral hemorrhage & $12(63.16)$ \\
Brain stem infarction & $1(5.26)$ \\
Malignant arrhythmia & $1(5.26)$ \\
Infection & $2(10.53)$ \\
Unconfirmed & $3(15.79)$ \\
\hline
\end{tabular}

significant. Interestingly, significantly more patients in the non-survival group received mechanical ventilation compared to the survival group $(13 / 19$ vs. $5 / 36, \mathrm{P}<0.05$; Table 6).

\section{Discussion}

Idiopathic TTP accounts for approximately 40-77\% of all TTP cases, and anti-ADAMTS13 autoantibodies can be detected in plasma samples from $90 \%$ of TTP patients at onset (17). In line with these data, in the present study, 39 patients $(70.91 \%)$ presented with idiopathic TTP. In addition, acquired TTP in the other patients was associated with autoimmune diseases, infections, tumor, pregnancy, and drugs. In this study, the 3 tumor patients presented with breast cancer, cervical cancer, and blood tumor, but the tumor was a previous history and not a recent history, therefore the direct correlation between tumor and TTP could not be determined.

The clinical manifestations of TTP varied and were complicated. Patients were usually hospitalized for various initial symptoms. The present study revealed that all 55 patients had thrombocytopenia combined with hemolysis, while 50 patients $(90.91 \%)$ presented with neurological abnormalities, including headache and vertigo (26, 47.27\%), aphasia $(6,10.91 \%)$, delirium $(9,16.36 \%)$, disturbance of consciousness (41, 75.55\%), and convulsion (28, 50.91\%). Not all patients simultaneously showed the TTP pentad

Table 5 Baseline characteristics of patients with acquired thrombotic thrombocytopenic purpura (mean \pm SD)

\begin{tabular}{|c|c|c|c|c|}
\hline Characteristic & Survivors & Non-survivors & t value & $P$ value \\
\hline APACHE II score & $11.50 \pm 4.49$ & $20.12 \pm 7.83$ & 5.173 & 0.000 \\
\hline SOFA score & $7.74 \pm 2.10$ & $12.06 \pm 3.27$ & 5.008 & 0.000 \\
\hline Platelet counts $\left(10^{9} / \mathrm{L}\right)$ & $13.26 \pm 10.07$ & $11.35 \pm 5.87$ & -0.727 & 0.471 \\
\hline Schistocytes (\%) & $3.44 \pm 3.13$ & $6.18 \pm 4.69$ & 2.183 & 0.035 \\
\hline HCT & $0.21 \pm 0.07$ & $0.18 \pm 0.04$ & 1.329 & 0.189 \\
\hline Total bilirubin ( $\mu \mathrm{mol} / \mathrm{L})$ & $58.62 \pm 41.34$ & $76.67 \pm 39.61$ & 1.482 & 0.144 \\
\hline Blood creatinine ( $\mu \mathrm{mol} / \mathrm{L})$ & $99.55 \pm 88.21$ & $84.98 \pm 41.54$ & -0.629 & 0.532 \\
\hline
\end{tabular}

APACHE II, Acute Physiology and Chronic Health Evaluation II; SOFA, sequential organ failure assessment; HCT, hematocrit; APTT, activated partial thromboplastin time; PEX, plasma exchange; SD, standard deviation. 
Table 6 Clinical characteristics of patients with acquired thrombotic thrombocytopenic purpura

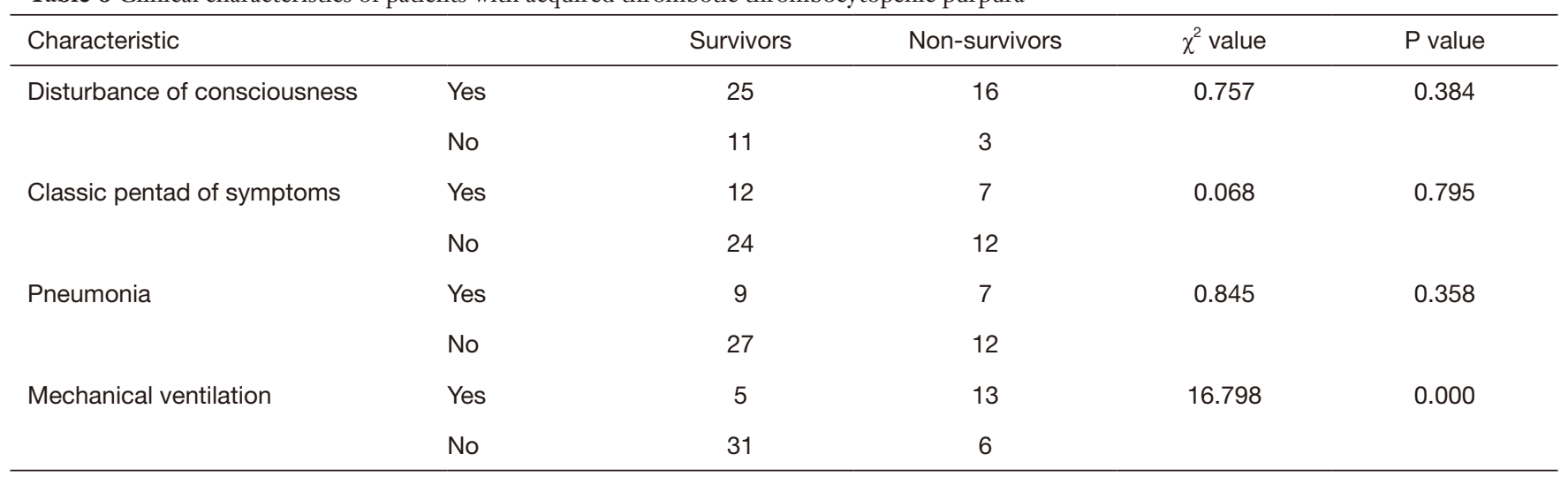

symptoms. In the present study, only 19 patients had the classic TTP pentad, while 5 patients only presented with 2 symptoms (thrombocytopenia and hemolysis). Therefore, if a patient presents with combined thrombocytopenia and intravascular hemolysis, especially in the presence of neurological abnormalities, the occurrence of TTP should be considered.

For the last few decades, PEX has been considered to be the most effective therapy for TTP patients, and has become a standard of care (18). PEX can supplement efficient ADAMTS13 activity, eliminate ultralarge von Willebrand factor (ULVWF), and remove anti-ADAMTS13 autoantibodies from the circulation. Since the introduction of PEX in TTP therapy in the 1970s, the overall mortality has been reduced from $90 \%$ to $10-20 \%$ (19). In the present study, the mortality of patients treated with PEX $(32.65 \%)$ was higher. One of the main reasons for the high mortality in the present study group was the severity of the cases due to diagnostic delays. The patients were referred to our hospital with advanced symptoms, and the delay between the onset of disease and initiation of treatment may have hampered the PEX efficacy. The second reason for the high mortality was the low dose of PEX caused by FFP shortages, with some patients only receiving 2,000 $\mathrm{mL}$ PEX daily. In the present report, the response rate was the highest in patients treated with PEX combined with corticosteroid and rituximab (81.25\%), and lowest in patients treated with pulse dose corticosteroid (50\%). Pulse corticosteroid therapy was also correlated with the highest rate of fatal infections $(n=2)$. However, these results were not statistically significant and no final conclusions can be drawn from this. Therefore, a larger study cohort, preferably in a prospective setting, is warranted.
In summary, the present retrospective analysis revealed the clinical characteristics and outcomes in patients with acquired TTP who were treated in a university hospital in China. The APACHE II and SOFA scores, as well as LDH levels and schistocytosis were higher in nonsurvivors compared to survivors, and more non-survivors required mechanical ventilation. These markers and scores were correlated to the severity of TTP and may be useful indicators for assessing the prognosis of TTP patients. However, due to the retrospective nature of this trial and the small size of the study group, it was not possible to draw a final conclusion. Further studies involving a larger patient cohort is warranted.

\section{Acknowledgments}

Funding: This work was supported by Gusu Health Talents Programme (No. GSWS2020006), Science Foundation of Jiangsu Commission of Health (H2018117) and Suzhou People's Livelihood Science and Technology Project (SYS201742).

\section{Footnote}

Reporting Checklist: The authors have completed the STROBE reporting checklist. Available at http://dx.doi. org/10.21037/apm-21-759

Data Sharing Statement: Available at http://dx.doi. org/10.21037/apm-21-759

Conflicts of Interest: All authors have completed the ICMJE uniform disclosure form (available at http://dx.doi. 
org/10.21037/apm-21-759). The authors have no conflicts of interest to declare.

Etbical Statement: The authors are accountable for all aspects of the work in ensuring that questions related to the accuracy or integrity of any part of the work are appropriately investigated and resolved. This study was reviewed and approved by the Ethics Committee of The First Affiliated Hospital of Soochow University (No. 2021115). All procedures performed in studies involving human participants were in accordance with the ethical standards of the institutional and national research committee and in accordance with Helsinki Declaration (as revised in 2013). Written informed consent was obtained from all participating patients.

Open Access Statement: This is an Open Access article distributed in accordance with the Creative Commons Attribution-NonCommercial-NoDerivs 4.0 International License (CC BY-NC-ND 4.0), which permits the noncommercial replication and distribution of the article with the strict proviso that no changes or edits are made and the original work is properly cited (including links to both the formal publication through the relevant DOI and the license). See: https://creativecommons.org/licenses/by-nc-nd/4.0/.

\section{References}

1. Scully M, Hunt BJ, Benjamin S, et al. Guidelines on the diagnosis and management of thrombotic thrombocytopenic purpura and other thrombotic microangiopathies. Br J Haematol 2012;158:323-35.

2. Terrell DR, Williams LA, Vesely SK, et al. The incidence of thrombotic thrombocytopenic purpura-hemolytic uremic syndrome: all patients, idiopathic patients, and patients with severe ADAMTS-13 deficiency. J Thromb Haemost 2005;3:1432-6.

3. George JN. Clinical practice. Thrombotic thrombocytopenic purpura. N Engl J Med 2006;354:1927-35.

4. Furlan M, Robles R, Galbusera M, et al. von Willebrand factor-cleaving protease in thrombotic thrombocytopenic purpura and the hemolytic-uremic syndrome. N Engl J Med 1998;339:1578-84.

5. Tsai HM, Lian EC. Antibodies to von Willebrand factorcleaving protease in acute thrombotic thrombocytopenic purpura. N Engl J Med 1998;339:1585-94.

6. Hassan S, Westwood JP, Ellis D, et al. The utility of
ADAMTS13 in differentiating TTP from other acute thrombotic microangiopathies: results from the UK TTP Registry. Br J Haematol 2015;171:830-5.

7. Sarig G. ADAMTS-13 in the Diagnosis and Management of Thrombotic Microangiopathies. Rambam Maimonides Med J 2014;5:e0026.

8. Han B, Page EE, Stewart LM, et al. Depression and cognitive impairment following recovery from thrombotic thrombocytopenic purpura. Am J Hematol 2015;90:709-14.

9. Lewis QF, Lanneau MS, Mathias SD, et al. Long-term deficits in health-related quality of life after recovery from thrombotic thrombocytopenic purpura. Transfusion 2009;49:118-24.

10. Hughes C, McEwan JR, Longair I, et al. Cardiac involvement in acute thrombotic thrombocytopenic purpura: association with troponin $\mathrm{T}$ and $\mathrm{IgG}$ antibodies to ADAMTS 13. J Thromb Haemost 2009;7:529-36.

11. Deford CC, Reese JA, Schwartz LH, et al. Multiple major morbidities and increased mortality during long-term follow-up after recovery from thrombotic thrombocytopenic purpura. Blood 2013;122:2023-9; quiz 2142.

12. Patschan D, Witzke O, Dührsen U, et al. Acute myocardial infarction in thrombotic microangiopathies-clinical characteristics, risk factors and outcome. Nephrol Dial Transplant 2006;21:1549-54.

13. George JN. Corticosteroids and rituximab as adjunctive treatments for thrombotic thrombocytopenic purpura. Am J Hematol 2012;87 Suppl 1:S88-91.

14. Zhou Z, Nguyen TC, Guchhait P, et al. Von Willebrand factor, ADAMTS-13, and thrombotic thrombocytopenic purpura. Semin Thromb Hemost 2010;36:71-81.

15. Yu F, Tan Y, Zhao MH. Lupus nephritis combined with renal injury due to thrombotic thrombocytopaenic purpura-haemolytic uraemic syndrome. Nephrol Dial Transplant 2010;25:145-52.

16. Sam C, Desai P, Laber D, et al. Pegylated bovine carboxyhaemoglobin utilisation in a thrombotic thrombocytopenic purpura patient. Transfus Med 2017;27:300-2.

17. Reese JA, Muthurajah DS, Kremer Hovinga JA, et al. Children and adults with thrombotic thrombocytopenic purpura associated with severe, acquired Adamts13 deficiency: comparison of incidence, demographic and clinical features. Pediatr Blood Cancer 2013;60:1676-82.

18. Rock GA, Shumak KH, Buskard NA, et al. Comparison of plasma exchange with plasma infusion in the treatment 
of thrombotic thrombocytopenic purpura. Canadian Apheresis Study Group. N Engl J Med 1991;325:393-7.

19. Bandarenko N, Brecher ME. United States Thrombotic Thrombocytopenic Purpura Apheresis Study Group (US TTP ASG): multicenter survey and retrospective analysis

Cite this article as: Huang F, Li XP, Xu Y, Li XY, Li DR, Hao $\mathrm{Y}$, Yu ZQ, Jin J, Wang J. Clinical characteristics and outcomes of adult patients with acquired thrombotic thrombocytopenic purpura: a single center retrospective study. Ann Palliat Med 2021;10(5):5351-5358. doi: 10.21037/apm-21-759 of current efficacy of therapeutic plasma exchange. J Clin Apher 1998;13:133-41.

(English Language Editor: J. Teoh) 